\title{
Aerodynamic Analysis of Three-Section Flapping Wing by Numerical Simulation
}

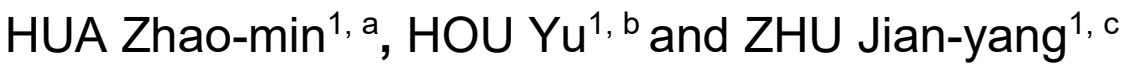 \\ ${ }^{1}$ Key Laboratory of Metallurgical Equipment and Control Technology, Wuhan University of Science \\ and Technology, Hubei Wuhan 430081, China \\ a289646316@qq.com, bhouyu@wust.edu.cn, czhujy@wust.edu.cn
}

\begin{abstract}
Keywords: three-section flapping wing; wing folding; kinematic model; aerodynamic model; lift drag ratio

Abstract. Three-section flapping wing is a new kind of bionic wing, which had better bionic performance compared to existing single-section and two-section wings. In order to realize it, on the basis of single drive mode, a novel mechanism was designed that can realize both flapping and double folding motions. Based on it, analytical and aerodynamic models of three-section flapping wing mechanism were established. Three kinds of typical flight conditions were selected to calculate the aerodynamic changes of three-section and two-section wings. The results show that the mechanism meets the requirements of bionic design. Compared with other flapping wings, the three-section flapping wing mechanism can achieve dexterous and efficient bionic movements and also has better bionic kinetic characteristics and aerodynamic performance.
\end{abstract}

\section{Introduction}

The bionic flapping wing aircraft is a new conceptual aircraft designed across multiple disciplines. It has the characteristics of light quality, strong concealment, small noise and good stability[1]. At the same time, flapping wing aircraft has a broad application prospect in various fields, such as national defense and civil service. Therefore, it is of great significance for the research of flapping wing aircraft.

In flapping wing aircraft, flapping wing mechanism is the core part of the performance of the aircraft. The simplest single-section flapping wing mechanism can not fold and can only achieve the basic flapping motion, including the single crank double rocker mechanism[2] and so on. The existing single-section flapping wing mechanisms have many problems, such as asymmetric motion process[3], poor flight stability[4], poor bionics, etc.

In order to improve the flying performance of flapping wing mechanism, lots of researchers have carried out a series of studies to optimize the motion characteristics and aerodynamic characteristics of it. Two-section flapping wing mechanism can achieve single-folding motion, including the SmartBird[5] and BionicOpter[6] of Festo company, Joseph Bahlmand's bat-like flapping wing aircraft[7], etc. Duan designed an active deformed flapping wings with a steering engine to control folding [8]; Gu designed a new morphing wing[9]. They all proved that the two-section flapping wing had some advantages compared with the single-section flapping wing in the aerodynamic characteristics and bionics. The existing two-section flapping wing has preliminarily realized flapping, twisting and single-folding, which is more close to the flight performance of the birds in the bionics.

However, there have been few studies focused on folding[10]. As an important factor, the researches on bionics are not enough. The motions still can not imitate the flying characteristics of birds, and the flight flexibility and aerodynamic efficiency have been limited. Aiming at this problem, according to the flight characteristics of birds, a three-section flapping wing mechanism was designed that could achieve flapping and double-folding, its aerodynamic characteristics had been studied. 


\section{Analysis model of three-section flapping wing}

Analysis model of single-section and two-section flapping wing. At present, the most common single-section flapping wing[11] mainly represents flapping and twisting. The two-section flapping wing model[12] can achieve flapping, twisting and single-folding, as equation (1):

$$
\left\{\begin{array}{l}
\beta(t)=\beta_{0}+\beta_{\max } \sin \left[\omega(t) t+\varphi_{\beta}\right]-\delta(t) \frac{\operatorname{sgn}\left(l-b_{1}\right)+1}{2} \\
\alpha(t)=\alpha_{0}+\alpha_{\text {max }} \sin \left[\omega(t) t+\varphi_{\alpha}\right]
\end{array}\right.
$$

Where $\beta$ is flapping angle, $\alpha$ is twisting angle. $\delta(t)$ is the flapping deviation angle of inner and outer section of wings, as equation (2):

$$
\delta(t)=\pi-\gamma_{1} .
$$

Where $\gamma_{1}$ is folding angle of the wing, that is also the included angle of the inner and outer section of wings.

The two models above have defects in the description of large bird wings: Wings of birds include three sections, so the movements of wings can not be shown well in single-section and two-section models, especially the active folding of wings.

Three-section flapping wing model. In order to describe the movements of wings better, a three-section flapping wing is designed as shown in figure 1.

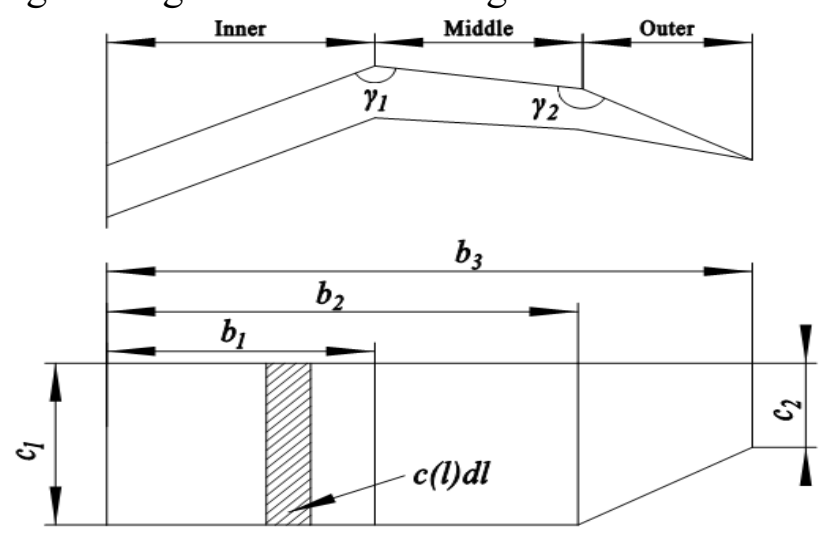

Fig.1 The shape of the three-section flapping wing

$\beta_{0}$ is the initial flapping angle, $\beta_{\max }$ is the maximum flapping angle, $\alpha_{0}$ is the initial twisting angle, $\alpha_{\max }$ is the maximum twisting angle, $l$ is the spanwise position of the flapping wing section, $b_{1}$ is the length of inner wing, $b_{2}-b_{1}$ is the length of middle wing, $b_{3}-b_{2}$ is the length of outer wing, and $\delta(t)$ is the folding angle function. So the three-section flapping wing model is worked out as equation (3):

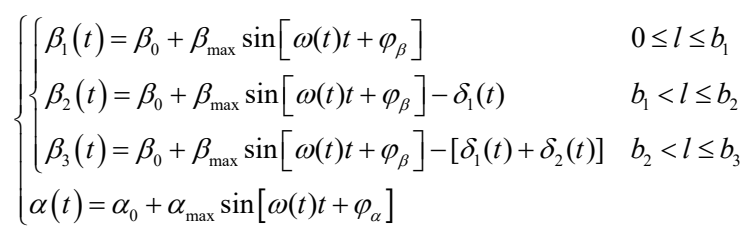

Where $\delta(t)$ is expressed as equation (4):

$$
\left\{\begin{array}{l}
\delta_{1}(t)=\pi-\gamma_{1} \\
\delta_{2}(t)=\pi-\gamma_{2} \\
\mathrm{~V} \gamma_{2}=c \mathrm{~V} \gamma_{1}
\end{array}\right.
$$


Where $\gamma_{1}$ is the first folding angle of the wing, that is also the included angle of the inner and middle section of wings. $\gamma_{2}$ is the second folding angle of flapping wing, that is also the included angle of the middle and outer section of wings. $c$ is the constant of the relationship between the first and second folding angles.

\section{Aerodynamic calculation model of flapping wing}

Flapping wing mechanism design.According to the flight characteristics of birds, the following design requirements are proposed for flapping wing mechanism [13]:

(1) The mechanism is required to achieve flapping and double-folding coupling motions, where flapping angle $\alpha \geq 60^{\circ}$ and folding angle $\gamma \geq 20^{\circ}$.

(2) The frequency of flapping and folding motion is $f=2 \mathrm{~Hz}$, that is, a complete flapping cycle $T=0.5 \mathrm{~s}$.

(3) The airfoil size of the mechanism is $180 \mathrm{~cm}$, and the maximum width of the body section is not more than $20 \mathrm{~cm}$.

(4) Wing motions are required to have symmetry and stability;

(5) The structure should be simple, and the flapping wing movements have the quick-return characteristic.

Based on the three-section flapping wing model above, a new mechanism that can realize flapping and double-folding is designed as shown in figure 2.

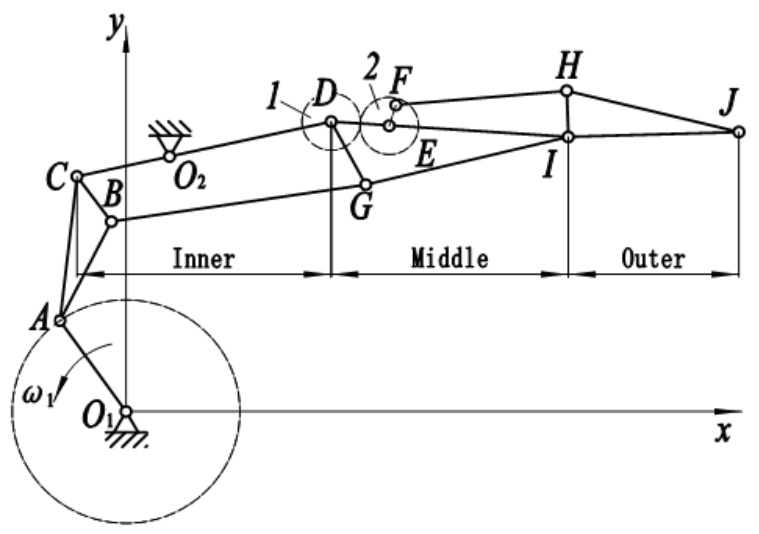

Fig.2 Flapping wing mechanism

In figure 2, point $O_{1}$ and point $O_{2}$ are the hinge points of flapping wing and frame, the crank $O_{1} A$ is the driving link. When the crank $O_{1} A$ rotates, $C D$ rotates around the point $O_{2}$, realizes the flapping of inner wing; $C D G B$ deforms and drives the rotation of middle wing, $B G$ 、 $G I$ rotates and achieve the first folding motion;

Gear 1 and gear 2 are fixed on point $D$ and point $E$, the gear 1 is hinged at point $D$, and fixed with link $C D$; the gear 2 is hinged at point $E$, and fixed with link $D I$. The relative angle change of the inner and middle section makes the gear 1 drive gear 2 to rotate, $H I$ rotates and drives $I J$, realizes the second folding motion.

The flapping mechanism meets the requirements of motions, achieves stable flapping and double-folding motions. The swooping-lifting process, as shown in figure 3, conforms to the flapping wing movement of the birds.

The wingspan of the designed mechanism is $180 \mathrm{~cm}$, the fuselage section width is $20 \mathrm{~cm}$. The inner wing $C D=31 \mathrm{~cm}$, the middle wing $D I=26 \mathrm{~cm}$ and the outer wing $H J=22 \mathrm{~cm}$ are initially designed.

Then link parameters is put into the model above, the motion angle $\beta(t)$ in a period of each section can be got as shown in figure 4 . The angle of the inner wing decreases slowly in $0 \sim 0.3 \mathrm{~s}$ (swooping stage) and increases rapidly in $0.3 \sim 0.5 \mathrm{~s}$ (lifting stage), which proves that the flapping motion has the quick return characteristic. 
At the same time, the first folding angle $\gamma_{1}=10.8^{\circ} \sim 75.6^{\circ}$, the second folding angle $\gamma_{2}=15.5^{\circ} \sim 26.2^{\circ}$. The motions conform the flight rule of birds and meet the design requirements mentioned above.

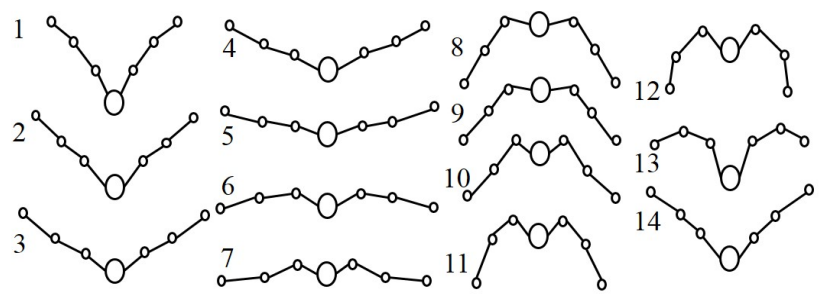

Fig. 3 The folding change of the wing in one period

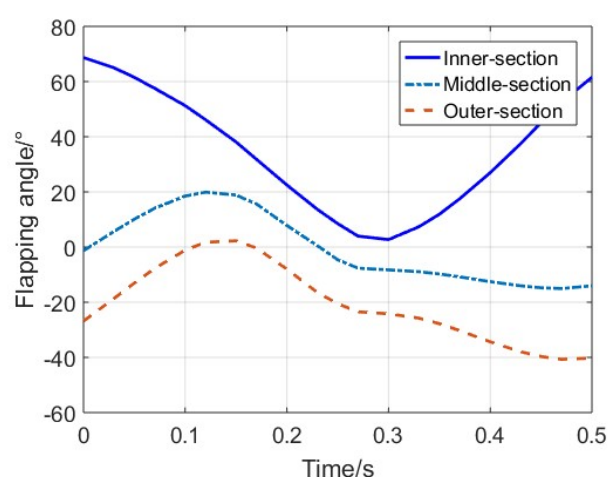

Fig.4 The change of the angles in one period

Aerodynamic model of flapping wing mechanism. In actual flight process, compared to twisting and swinging [14], flapping and folding have greater impact on flight lift. The aerodynamic model of three-section wing is presented in this section.

The aerodynamic model of three-section wing is established: horizontal velocity $v_{\infty}$, initial attack angle $\alpha_{0}$. The average aerodynamic force can be obtained by calculating the aerodynamic force of flapping wing spanwise micro section $c(l) d l$ (Figure. 1) and integrating of periodic time and length. The actual airflow angle of the flapping wing extension can be expressed as equation (5)

$$
\alpha(t)=\alpha_{0}-\arctan \left(\frac{v_{f}}{v_{\infty}}\right) .
$$

Where $v_{f}$ is the flapping speed of the micro section, $\arctan \left(\frac{v_{f}}{v_{\infty}}\right)$ is the airflow angle of the flapping. The average aerodynamic force in one period can be worked out as equation (6) by integrating the micro section.

$$
\left\{\begin{array}{l}
L=\frac{2}{T} \int_{0}^{T} \int_{0}^{b_{3}} q\left\{C_{l} \cos \left[\alpha(t)-\alpha_{0}\right]+C_{d} \sin \left[\alpha(t)-\alpha_{0}\right]\right\} \\
T=\frac{2}{T} \int_{0}^{T} \int_{0}^{b_{3}} q\left\{C_{l} \sin \left[\alpha(t)-\alpha_{0}\right]-C_{d} \cos \left[\alpha(t)-\alpha_{0}\right]\right\} c d l d t
\end{array}\right.
$$

Where the actual dynamic pressure of a flapping wing $q=q\left(v_{\infty}, \beta(t), l\right) . C_{l}, \quad C_{d}$ is the lift and drag coefficient: $C_{l}=C_{l}(\alpha), C_{d}=C_{d}(\alpha)$, they will be calculated by using XLFR5 software in later section. $\beta(t)$ is the flapping angle; Micro section length $c=c(l)$ is:

$$
c(l)=\left\{\begin{array}{cc}
c_{1} & \left(0 \leq l \leq b_{2}\right) \\
\frac{c_{1} b_{3}-b_{2} c_{2}-l\left(c_{1}-c_{2}\right)}{b_{3}-b_{2}} & \left(b_{2}<l \leq b_{3}\right)
\end{array}\right.
$$

The actual dynamic pressure $q\left[v_{\infty}, \beta(t), l\right]$ is related to the flight speed, flapping speed and the location of its direction.

$$
q\left[v_{\infty}, \beta(t), l\right]=\frac{1}{2} \rho|v|^{2}=\frac{1}{2} \rho\left|v_{\infty}+v_{f}\right|^{2} .
$$

$v_{\infty}$ is the horizontal speed and $v_{f}$ is the speed of the wing relative to the air. The actual speed $v$ is vector superposition of $v_{\infty}$ and $v_{f}$.

$$
v_{f}[\beta(t), l]=\omega[\beta(t), l] l .
$$




\section{Aerodynamic calculation and analysis of flapping wing}

Lift calculation. The aerodynamic characteristics of flapping wing mechanism are closely related to its airfoil. In order to improve the aerodynamic performance, the airfoil that the front is thick and has large camber is selected for three-section flapping wing. Import this kind of airfoil into XFLR5 software.

The actual wind speed is $6 \mathrm{~m} / \mathrm{s}-22 \mathrm{~m} / \mathrm{s}$. Define the wind velocity is $10 \mathrm{~m} / \mathrm{s}$. When the temperature is $20^{\circ} \mathrm{C}$, the kinetic viscosity coefficient $v=1.5 \times 10^{-5} \mathrm{~m}^{2} / \mathrm{s}$ and Reynolds number range is $1 \times 10^{4}<\operatorname{Re}<5 \times 10^{5}$. Define Reynolds number as $\operatorname{Re}=10^{5}$, air density $\rho=1.225 \mathrm{~g} / \mathrm{m}^{3}$, horizontal velocity $\left|\nu_{\infty}\right|=5 \mathrm{~m} / \mathrm{s}$ and initial attack angle $\alpha_{0}=3^{\circ}$.

Use the XFLR5 software to calculate the lift and drag coefficients of the airfoil at different attack angles, as shown in figure 5.

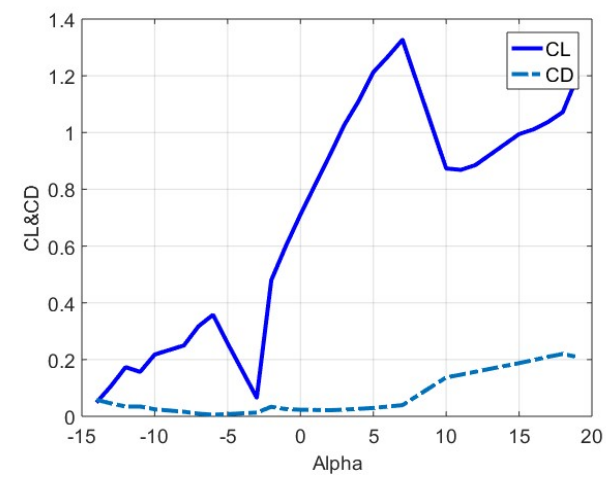

(a)The lift coefficient CL and the drag coefficient CD

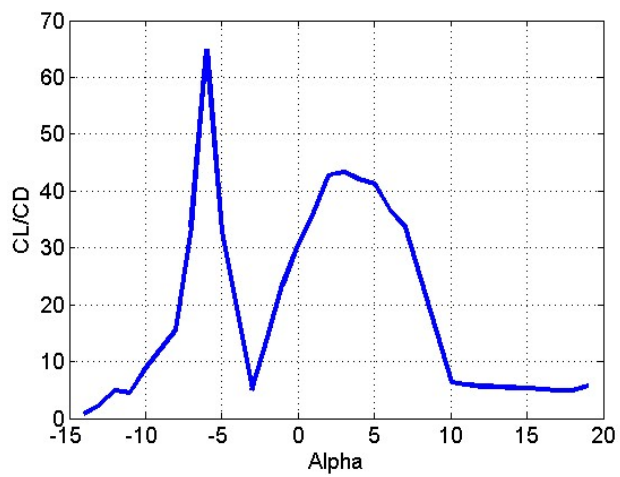

(b)The lift-drag ratio $\mathrm{CL} / \mathrm{CD}$

Fig.5 Aerodynamic coefficient curves of airfoil

According to the model above, the lift-drag ratio is the largest when $\alpha=3^{\circ}$ in the range of $\alpha \geq 0^{\circ}$. Without considering the influence of attack angle on lift, assume the attack angle is always $\alpha=3^{\circ}$, the lift change curve in one period of the three-section flapping wing is obtained, as shown in figure 6.

The lift value of three-section flapping wing is higher than the two-section flapping wing, and the maximum value is increased by $5.5 \%$, which shows its superiority.

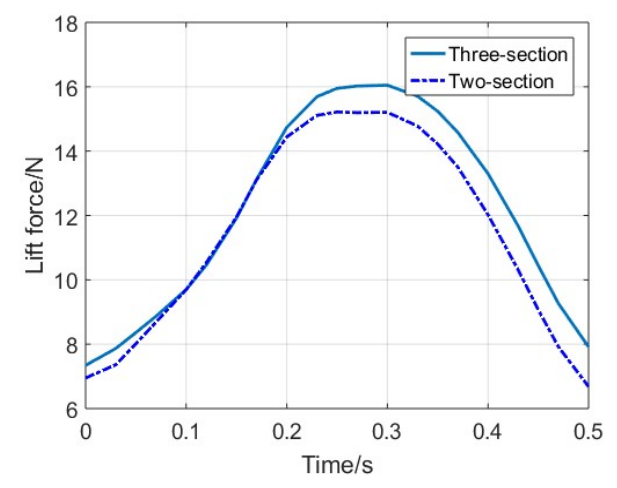

Fig.6 The lift change in one period

Analysis of aerodynamic characteristics under different flight states. In changeable environments, birds can keep good flight performance under different flight states. Figure 7 shows a typical flight state, so three critical states are selected as the target [15] of flight performance optimization: climbing, diving and cruising. 


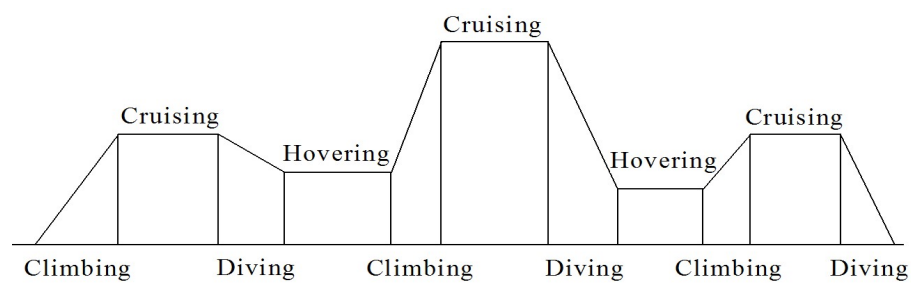

Fig.7 Flowchart of typical bird flight

In the simulation, the vortex lattice method (VLM) is used as the analysis method, and the aerodynamic force of the three-section wing in different states is analyzed by using the XFLR5 software. According to the flight conditions, the flow velocity $v=10 \mathrm{~m} / \mathrm{s}$, and there is no sideslip in the process.

\section{(1)Climbing state}

The state that birds get lift at take-off is called climbing state. In the course of climbing, it is necessary to get a larger lift-drag ratio.

During the climbing state, when the flapping angle is increased from $\beta_{1}=0^{\circ}$ to $\beta_{1}=20^{\circ}$, the attack angle is increased from $\beta_{1}=0^{\circ}$ to $\beta_{1}=20^{\circ}$ synchronously.

The wing angle in the three-section flapping wing is $\beta_{1}=0^{\circ} \sim 20^{\circ}, \beta_{2}=-7.97^{\circ} \sim 11.68^{\circ}, \beta_{3}=-23.83^{\circ} \sim-32.33^{\circ}$, as shown in figure 4 . In the two-section flapping wing, $\beta_{1}^{\prime}=0^{\circ} \sim 20^{\circ}, \beta_{2}^{\prime}=-7.97^{\circ} \sim 11.68^{\circ}$.

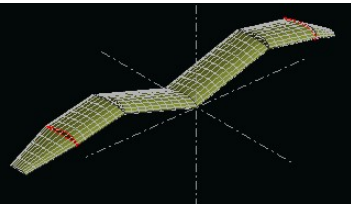

(a)three-section climbing layout

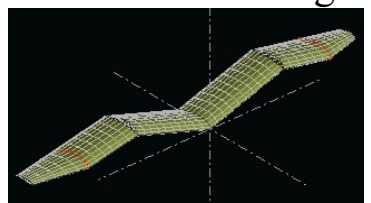

(b)two-section climbing layout

Fig.8 Climbing state layout

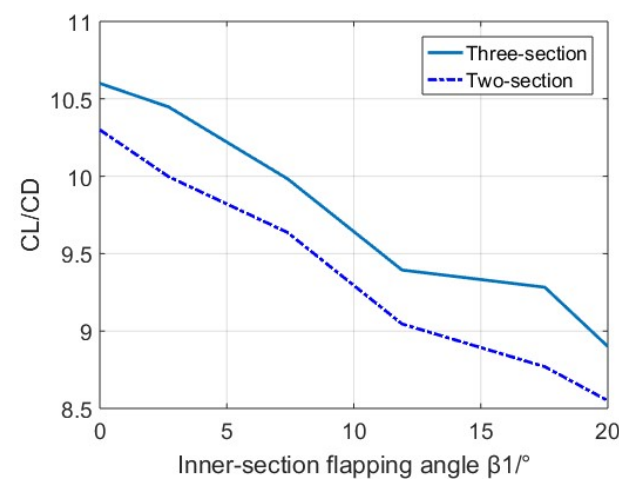

Fig.9 The lift-drag ratio in climbing state

In climbing state, the flapping angle of the inner wing gradually increases from $0^{\circ}$ to $20^{\circ}$. In this process, the lift-drag ratio of the three-section flapping wing is greater than that of the two-section flapping wing.

(2)Cruising state

In cruising state, large birds are in flight stability and depend on their wings to glide. The cruising state saves energy most during the entire flight. The drag is small and the efficiency is high in this state.

In cruising state, the wing angle in the three-section flapping wing is $\beta_{1}=0^{\circ}, \beta_{2}=-7.97^{\circ}, \beta_{3}=-23.83^{\circ}$, as shown in figure 4. In the two-section flapping wing, $\beta_{1}^{\prime}=0^{\circ}, \beta_{2}{ }_{2}=-7.97^{\circ}$. The attack angle is $\alpha=-5^{\circ} \sim 5^{\circ}$.

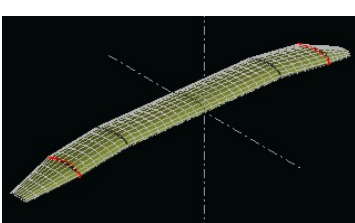

(a)three-section cruising layout

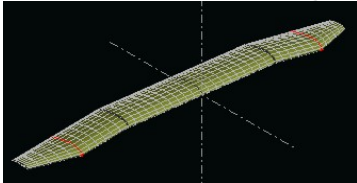

(b)two-section cruising layout

Fig.10 Cruising state layout

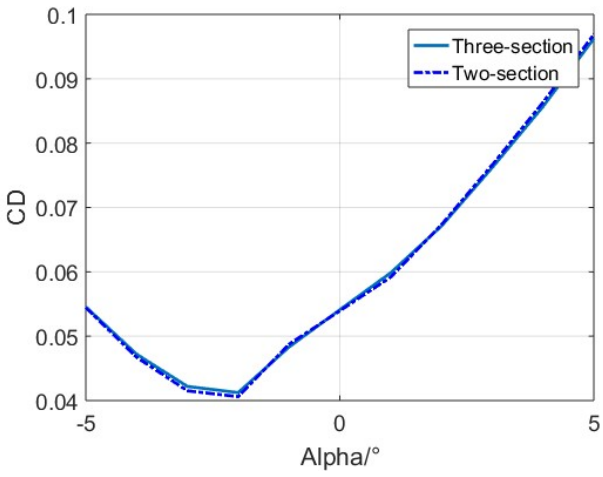

Fig.11 Drag coefficient CD in cruising state 
The effective attack angle of the cruising state is $-5^{\circ}<\alpha<5^{\circ}$. In this range, drag coefficient of the three-section wing is basically equal to the two-section wing. As shown in figure 10, the shape of the three-section and the two-section are basically the same, therefore their drag coefficients are also basically equal.

(3)Diving state

Diving state is that birds are hunting or landing. This state does not require large lift and lift-drag ratio, but there should be small drag.

The shape of the wing is basically locked in diving state. The wing angle in the three-section flapping wing is $\beta_{1}=68.7^{\circ}, \beta_{2}=-1.31^{\circ}, \beta_{3}=-26.83^{\circ}$, as shown in figure 4 . In the two-section flapping wing, $\beta_{1}^{\prime}=68.7^{\circ}, \beta_{2}^{\prime}=-1.31^{\circ}$. The attack angle is $\alpha=-10^{\circ} \sim 0^{\circ}$.

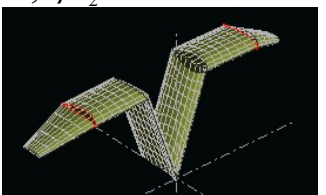

(a)three-section diving layout

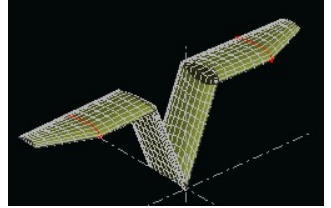

(b)two-section diving layout

Fig. 12 Diving state layout

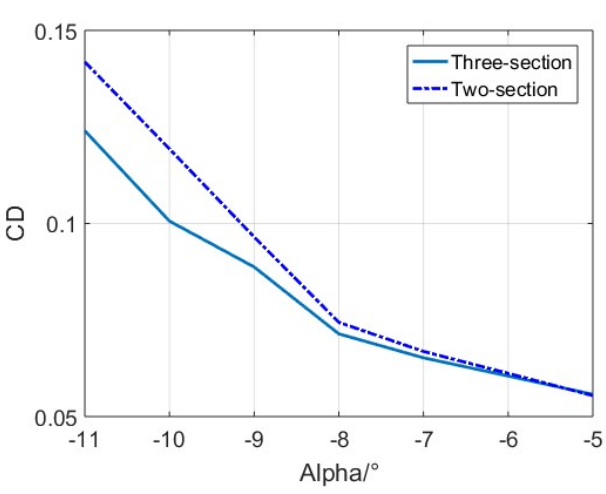

Fig.13 Drag coefficient in diving state

In diving state, the effective attack angle is $-15^{\circ}<\alpha<-5^{\circ}$, in this range the drag coefficient of three-section wing is less than the two-section wing, so the three-section wing performs better.

In the three kinds of flight states, the aerodynamic parameters of three-section wing and two-section wing are shown in table 1, it can be found that the lift-drag ratio is obviously increased, respectively, increased to about $3 \%, 2.88 \%$ and $11.53 \%$ in three different states, which proves that the three-section wing can achieve the better aerodynamic performance.

In the parameters of three typical states, the minimum drag coefficient under diving state of three-section is less than two-section, which proves that three-section flapping wing performs better in diving state; In cruising state the minimum drag coefficient is almost the same. In general, the flight performance of three-section flapping wing has been significantly improved in the typical flight states.

Table 1. Effective airfoil aerodynamic parameters in three states

\begin{tabular}{cccc}
\hline State & Parameters & Three-section wing & Two-section wing \\
\hline Climbing & Max lift-drag ratio & 10.61 & 10.30 \\
Cruising & Min drag resistance & 0.0402 & 0.0401 \\
Cruising & Max lift-drag ratio & 16.267 & 15.811 \\
Diving & Min drag resistance & 0.052 & 0.053 \\
Diving & Max lift-drag ratio & 7.804 & 6.997 \\
\hline
\end{tabular}

\section{Conclusions}

Aiming at the three-section model of the Bionic Flapping Wing, a new type of flapping wing mechanism is designed. The mechanism can simulate the flight posture of large birds and achieve flapping and double-folding motions.

Based on the aerodynamic analysis of the mechanism, a mathematical model of the three-section flapping wing mechanism is established, and the joint analysis is carried out for different geometric parameters, motion parameters and aerodynamic parameters. 
The aerodynamic performance of three-section flapping wing under typical flight states is analyzed, and compared with two-section flapping wing, it is proved that the three-section flapping wing has better aerodynamic and aerodynamic characteristics.

\section{Acknowledgements}

This work was financially supported by National Natural Science Foundation of China(51505347).

\section{References}

[1] Zhou QS.Design and Aerodynamic Analysis of a Bioinspired Flapping Wing Air Vehicle [D]. Harbin Institute of Technology, 2014(in Chinese)

[2] Jahanbin Z, Ghafari A S, Ebrahimi A, et al. Multi-body simulation of a flapping-wing robot using an efficient dynamical model[J]. Journal of the Brazilian Society of Mechanical Sciences \& Engineering, 2015, 38(1):1-17

[3] Dong EB. Research on Realization Mechanism and Some Key Technologies of Smart Morphing Aircraft Structures [D]. University of Science and Technology of China, 2010(in Chinese)

[4] XU YC,ZONG GH,et al. BI Shu-sheng Design and analysis of a spatial crank-rocker flapping-wing mechanism [J]. Journal of Aerospace Power,2009,24(1):204-208(in Chinese)

[5] Mackenzie D. Avionics. A flapping of wings [J]. Science, 2012, 335(6075):1430

[6] Gaissert N, Mugrauer R, Mugrauer G, et al. Inventing a Micro Aerial Vehicle Inspired by the Mechanics of Dragonfly Flight[M]// Towards Autonomous Robotic Systems. Springer Berlin Heidelberg, 2013:90-100

[7] Bahlman J W,Swartz S M,Breuer K S. Design and characterization of a multi-articulated robotic bat wing[EB/OL] . (2013). http://iopscience.Iop.org/1748-3190/8/1/016009/

[8] DUAN WB,ANG HS,XIAO TH.Design and wind tunnel testing of an active morphing wing ornithopter[J].Acta Aeronautica et Astronautica Sinica,2013,34(3):474-486(in Chinese)

[9] Gu LL.A Thesis in Measurement and Testing Technology \& Instruments[D].Nanjing University of Aeronautics and Astronautics,2013(in Chinese)

[10]MA N, He GP. The Simulation and Aerodynamic Analysis of Flapping-Wing MAV [J]. Science \& Technology Information, 2013, 33:1-2(in Chinese)

[11]ANG HS, ZENG R, DUAN WB, et al. Aerodynamic experimental investigation for mechanism of lift and thrust of flexible flapping-wing MAV[J]. Journal of Aerospace Power, 2007, 22(11):1938-1845(in Chinese)

[12]HUANG MY, XIAO TH, ANG HS. Design of an ornithopter with multisection flexible morphing wings [J]. Journal of Aerospace Power, 2016, 31(08):1838-1844(in Chinese)

[13]RUAN LH. Structure Design and Kinematic Research of Bionic Flapping-wing Flying Robot [D]. Wuhan University of Science and Technology, 2016(in Chinese)

[14] WANG KQ, CHEN SJ, LIU Y, et al. Design of Twisting Mechanism of Flapping-Wing Micro Air Vehicle [J]. Journal of Xi'an Technological University, 2015, (2): 125-129.DOI:10.16185 /j.jxatu.edu.cn.2015.02.007(in Chinese)

[15]Hao YF.Simulation Research on Morphing Wing Based on Sea Gull Morphology [J]. Computer Simulation, 2013, 30(09):36-40. (in Chinese) 